\title{
Idiotypic Sensitization In Utero of Children Born to Mothers with Schistosomiasis or Chagas' Disease
}

\author{
S. M. Eloi-Santos," E. Novato-Silva, ${ }^{*}$ V. M. Maselli, ${ }^{*}$ G. Gazzinelli, ${ }^{*}$ D. G. Colley, ${ }^{5}$ and R. Correa-Oliveira ${ }^{\ddagger}$

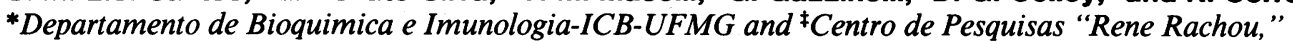 \\ FIOCRUZ 30190, Belo Horizonte, MG Brazil; and ${ }^{\S}$ Veterans Administration Medical Center \\ and Vanderbilt University School of Medicine, Nashville, Tennessee 37212
}

\begin{abstract}
Cord blood mononuclear cells (CBMC) of neonates born of mothers with Chagas' disease or schistosomiasis exhibited strong proliferative responses against idiotypes expressed on antibodies with specificity for Trypanosoma cruzi or Schistosoma mansoni antigens, respectively. These immunoaffinitypurified preparations were stimulatory if they were prepared from pools of patients' sera or from the mother's own serum, taken early during her pregnancy. These CBMC did not respond to normal immunoglobulin, and CBMC of neonates born of uninfected mothers did not respond to antibodies against either $T$. cruzi or $S$. mansoni, or normal immunoglobulin preparations.

We propose that in utero exposure of a fetus to some idiotypes expressed on placentally transferred antibodies induces anti-Id $T$ lymphocyte sensitization, which we detect as a proliferative response by CBMC exposed to immunoaffinity-purified antibodies expressing the relevant idiotypes. This is the first experimental evidence that children born of mothers with chronic infections undergo natural in utero idiotypic manipulations and are born possessing cellular anti-Id reactivity.
\end{abstract}

\section{Introduction}

Id and anti-Id manipulations of experimental hosts during the perinatal period have shown that the immunologic repertoire of the offspring can be profoundly influenced in utero or shortly after birth by either maternal or exogenously administered Ids or anti-Ids (1-7). In chronic, endemic infections with high levels of prevalence during the child-bearing years, such as schistosomiasis and Chagas' disease, active antibody production by infected, gravid women leads to considerable placental transfer of antibodies against the antigens of the infecting organism (8-10). We have predicted (11) that the Ids expressed on these transferred maternal antibodies could influence the responsiveness and subsequent morbidity expressed by their children, if they eventually become infected.

It is often observed in schistosomiasis and other chronic, endemic infections, that when people from nonendemic areas

Address reprint requests to Dr. Daniel G. Colley, Room F324, ACRE Building, Veterans Administration Medical Center, Nashville, TN 37212.

Received for publication 23 May 1989.

The Journal of Clinical Investigation, Inc

Volume 84, September 1989, 1028-1031 enter endemic areas and become infected, they are much more likely to develop severe pathologic consequences than people born and raised in an endemic area (11-14). Theoretically, if modulatory systems were induced in utero that could control immunopathogenesis, they could develop anamnestically upon infection of a child born of an infected mother, and contribute to the regulation of morbidity in endemic populations. Such maternally induced immunoregulatory alterations would be lacking in those born outside of an endemic area, to uninfected mothers.

We have used an in vitro proliferative assay to detect the presence of anti-Id $\mathrm{T}$ cells in the blood of patients with either or both schistosomiasis and Chagas' disease (15-19). In this system, the appropriate $\mathrm{T}$ cells proliferate in response to immunoaffinity-purified or monoclonal $(20,21)$ antibodies, against Schistosoma mansoni-soluble egg antigens (SEA) ${ }^{1}$ or T. cruzi epimastigote antigens (EPI) $(15,17,18)$. PBMC from individuals who have never had these infections are unresponsive to these antibodies, and neither patients with these infections nor uninfected subjects respond in culture to preparations of normal human immunoglobulin. We have further shown that the Ids on anti-SEA antibodies that are stimulatory in these cultures are cross-reactive Ids (CRIs) shared by antiSEA antibodies from mice with $S$. mansoni (Montesano, M. A., G. L. Freeman, Jr., G. Gazzinelli, and D. G. Colley, manuscript in preparation), which can be detected by rabbit anti-Id sera (19). Furthermore, in patients, these T cell-stimulatory CRIs are almost exclusively expressed on anti-SEA antibodies from people with the "intestinal," largely asymptomatic, clinical form of schistosomiasis (19).

We now demonstrate that, at birth, cord blood mononuclear cells (CBMC) from children born of mothers with these chronic, endemic infections respond to Ids on either anti-SEA or anti-EPI antibodies if (and only if) their mother was infected with $S$. mansoni or $T$. cruzi, respectively, during their gestation.

\section{Methods}

Patients. Pregnant women with active $S$. mansoni or Chagas' disease, or free of parasitologic or serologic evidence of past or present infections with $S$. mansoni or $T$. cruzi were studied during their pregnancies at prenatal clinics in Belo Horizonte, Brazil. Cord bloods were obtained from their children at birth. Informed consent was obtained

1. Abbreviations used in this paper: CBMC, cord blood mononuclear cells; CRI, cross-reactive idiotypes; EPI, T. cruzi epimastogote antigens; SEA, Schistosoma mansoni-soluble egg antigen. 
before the inclusion of each subject in the study, and patients with active schistosomiasis were offered appropriate chemotherapy for their infection after delivery, when treatment was no longer contraindicated.

Antigens and antibodies. The methods of preparation of the saline extracts SEA from $S$. mansoni eggs $(22,23)$ and EPI from $T$. cruzi epimastigotes $(17,24)$ has been detailed elsewhere. Likewise, the affinity purification of anti-SEA (15) and anti-EPI (17) antibodies on columns of SEA or EPI coupled to Sepharose 4B (Sigma Chemical Co., St. Louis, MO) from the sera of patients with schistosomiasis or Chagas' disease, respectively, has been presented. The antibody preparations used in this study were purified either from pools of sera from 12-15 intestinal schistosomiasis patients (19; anti-SEA), 12-15 patients with the cardiac form of Chagas' disease (17; anti-EPI), or from the individual sera of pregnant patients either early or late in their pregnancy.

Cell proliferation assays. CBMC-proliferative assays in response to preparations of anti-SEA $(40 \mu \mathrm{g} / \mathrm{ml}$ culture), anti-EPI antibodies ( 40 or $60 \mu \mathrm{g} / \mathrm{ml}$ culture), or normal human immunoglobulin $(40-120 \mu \mathrm{g} / \mathrm{ml}$ culture; Sigma Chemical Co.), or PHA ( $2 \mu \mathrm{l} / \mathrm{ml}$ culture; Difco Laboratories, Detroit, MI) were done as previously reported for PBMC (15, $17,18)$. Briefly, $1.5 \times 10^{5} \mathrm{CBMC}$ were cultured in triplicate cultures in flat-bottom microtiter wells (Titertek; Flow Laboratories, Rockville, MD) in medium consisting of $90.4 \%$ RPMI $1640,1.6 \%$ L-glutamine (stock $200 \mathrm{mM}$ ), 3\% antibiotic-antimycotic (stock 10,000 U penicillin, $10 \mathrm{mg}$ streptomycin, $25 \mu \mathrm{g}$ Fungizone/ml; Gibco Laboratories, Grand Island, NY) and 5\% heat-inactivated normal AB+ human serum. Cultures were maintained for 4 or $5 \mathrm{~d}$, labeled with $0.5 \mu \mathrm{Ci}$ per culture tritiated thymidine $(6.7 \mathrm{Ci} / \mathrm{mM} \mathrm{Sp}$ act; New England Nuclear, Boston, MA), and collected for scintillation counting $18 \mathrm{~h}$ later. Data were calculated as mean counts per minute of triplicate cultures, and are presented as experimental counts per minute - control (medium control) counts per minute $(E-C)$ values.

\section{Results}

Responses of CBMC to antibody pools, normal immunoglobulin, or PHA. The data presented in Fig. 1 show that CBMC of neonates born of mothers with either schistosomiasis or Chagas' disease consistently responded upon exposure to antiSEA or anti-EPI antibodies, respectively, isolated from pooled sera from patients with these infections. CBMC of newborns whose mothers were uninfected, and almost certainly had

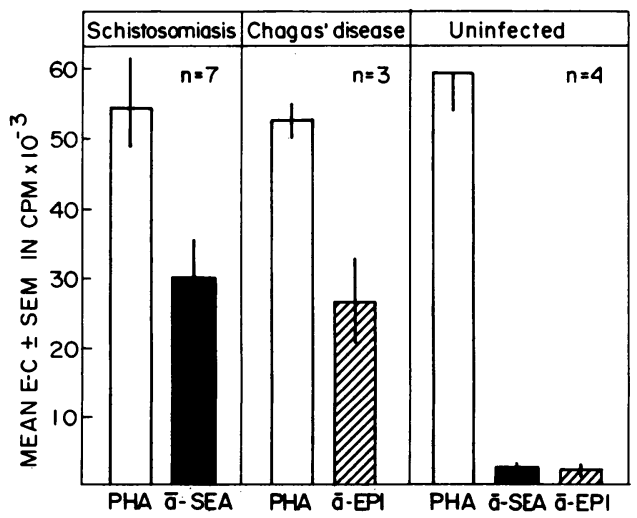

Figure 1. Mean proliferative responses of CBMC from neonates born of mothers with either schistosomiasis, Chagas' disease, or uninfected, upon exposure to PHA ( $\square$ ), anti-SEA antibodies from the pooled sera of intestinal, nonpregnant schistosomiasis patients $(\square)$, or anti-EPI antibodies from the pooled sera of cardiac, nonpregnant chagasic patients ( $($ ) $)$. The mean \pm SE control (unstimulated) counts per minute of the $14 \mathrm{CMBC}$ cultures $=1,767 \pm 327$. never been infected (by virtue of serologic data and their being from nonendemic areas and/or a more affluent socioeconomic group) were unresponsive to either the anti-SEA or anti-EPI antibody preparations (Fig. 1). CBMC cultures exposed to parallel or higher concentrations of normal human immunoglobulin preparations (Sigma Chemical Co.) did not proliferate (mean $\mathrm{E}-\mathrm{C} \mathrm{cpm}$ value $=1,113$; range $=53-3,559)$. CBMC from all neonates responded comparably to PHA regardless of whether their mothers had schistosomiasis or Chagas' disease or were uninfected (Fig. 1).

$C B M C$ responses to antibodies from their mother's sera. The CBMC of a neonate born of a mother with schistosomiasis or Chagas' disease responded to anti-SEA or anti-EPI antibodies, respectively, when the antibodies were isolated from their mother's serum obtained early in the pregnancy. In some cases, the CBMC were unresponsive to antibodies of the same general specificities (i.e., anti-SEA or anti-EPI) if they had been purified from the sera of individual patients other than their mother (Fig. 2). When affinity-purified antibodies prepared from pools of sera from nonpregnant patients $(15,17,19)$ were used (as in Fig. 1), this exclusivity was avoided (Fig. 2, patient B; Fig. 3, patient $\mathbf{H}$ ).

Preliminary data indicate that anti-SEA antibodies prepared from sera taken from gravid women in the latter half to two-thirds of their pregnancy often failed to stimulate CBMC from even their own newborns (Fig. $3 ; A-D, I$ ), or stimulated lesser responses than did pooled anti-SEA antibodies (Fig. 3; $E$ and $F$ ). As presented above, antibodies derived from a mother's serum early in her pregnancy, were clearly stimulatory for her neonates CBMC (Figs. 2 and 3; $G-I$ ). In the two cases studied thus far, where we were able to obtain antibody preparations from a gravid patient at two time points, the maternal antibodies from early in pregnancy stimulated a CBMC response, whereas those from later in pregnancy did not (Fig. 3; $H$ and $I$ ).

\section{Discussion}

The data presented here provide the first evidence that children born of mothers with chronic infections have, at birth, anti-Id cellular responses related to the specificities of their mother's infection. CBMC from these neonates proliferate specifically in response to appropriate anti-SEA or anti-EPI antibodies, and not to normal immunoglobulin preparations. The same anti-SEA and anti-EPI antibodies are not stimulatory for CBMC from neonates born of mothers without these chronic infections.

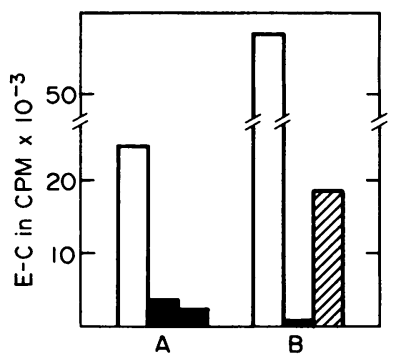

Figure 2. CBMC responses of a neonate born of a mother with Chagas' disease $(A)$ and CBMC responses of a neonate born of a mother with $S$. mansoni $(B)$. The potential stimulating antibodies, anti-EPI and anti-SEA, respectively, were purified from serum obtained early in the pregnancy of the neonate's own mother ( $\square$ ), or other individual patients $(\boldsymbol{\square})$, or

the pooled sera of intestinal, nonpregnant patients ( $(a)$. Data are given as $\mathrm{E}-\mathrm{C}$ counts per minute values. 


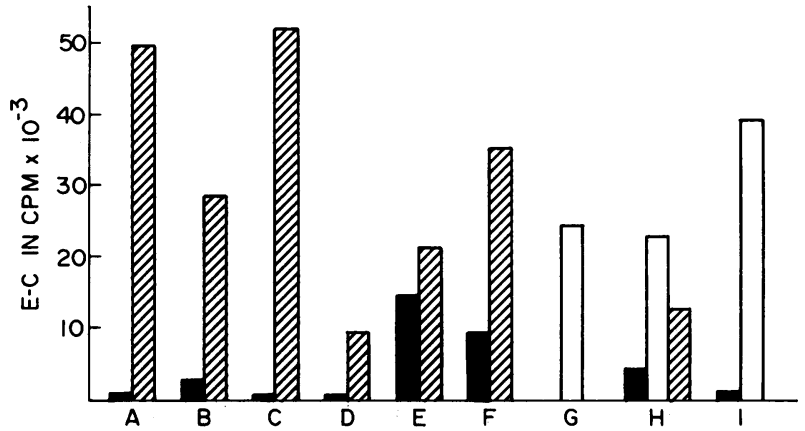

Figure 3. CBMC responses upon culture and exposure to anti-SEA or anti-EPI antibodies from their own mother's serum taken during the latter half of the second or third trimesters $(\square)$ or the first trimester (ㅁ) of pregnancy, or from pools of patients' sera as in Figs. 1 and 2 (ש). CBMC were from eight neonates born of mothers with $S$. mansoni $(A-H)$, and one born of a mother with Chagas' disease (I).

The consequences of this in utero idiotype-specific sensitization are unknown, and longitudinal studies of these chronic infections to discern possible effects are not feasible. In schistosomiasis, such studies would be unethical (due to the availability of applicable chemotherapeutic drugs). In Chagas' disease, they would require decades to observe disease progression. It is possible that in utero exposure and sensitization to these Ids could presensitize the offspring to antigens of the organisms (6). Furthermore, circulating antigens (25) of the organisms could also cross the placenta or be presented to newborns in their mother's milk (26), and contribute to the induction of neonatal responsiveness. Both the in utero induction of responsiveness (27-29) and unresponsiveness (30-31) to parasite antigens in offspring of mothers with experimental and/or clinical chronic helminthic infections have been reported. We have also observed in our ongoing studies of maternal schistosomiasis and Chagas' disease, the CBMC of children born of infected mothers often respond to the antigenic preparations SEA or EPI, respectively (Novato-Silva, E., G. Gazzinelli, and D. G. Colley, manuscript in preparation).

Children born with antigen and/or anti-Id responsive $T$ cells may have established a regulatory, suppressor immune response mode to certain antigens of the organisms with which their mother was infected. In murine malaria, this situation can be mediated through maternal IgG induction of specific $T$ suppressor cells in mice born to mothers immune to malaria (32) and prevents their subsequent immunization. Such in utero exposure could also affect an individual's response to eventual prophylactic immunization against the organism in a positive manner $(6,33)$. Vaccine development strategies should consider the potential differences that a given vaccine might have on endemic vs. nonendemic populations.

The initial data reported here on apparent idiotypic differences between antibodies of the same general specificities based on the time during pregnancy at which they were isolated, are very intriguing. These are obviously still operational, preliminary findings for which we do not yet have a satisfactory explanation. However, the less stimulatory antibodies, obtained later in pregnancy, do appear to serve as an unexpected negative control. By their inability to stimulate CBMC, they demonstrate that neonatal CBMC do not proliferate indescriminately upon exposure to all Ids or Ig preparations. It remains theoretically possible that the CBMC do interact with the antibody preparations from late in pregnancy, but the resultant response leads to a suppressed and therefore undetected, regulated state. However, the induction of differential regulatory circuitries by antibody preparations from different times during pregnancy, or any proposed differential regulation of given idiotypes during the progression of pregnancy, would be highly speculative. Only additional studies of these preliminary operationally defined results will allow conclusions about the differences that lead to this apparent phenomenon.

These data demonstrate that neonatal CBMC can respond to appropriate antibodies that are specific for antigens of organisms with which their mothers are chronically infected. Based on reports of the experimental effects of perinatal idiotypic manipulations $(1-7,32,33)$, this in utero idiotypic exposure and sensitization could be expected to at least lead to idiotypically different immunological responses in the children of infected mothers compared with children of uninfected mothers. This influence might be expressed perinatally (as shown here) and/or anamnestically, upon later infection.

\section{Acknowledgments}

We are grateful to Dr. S. J. Stewart for his critical readings of the manuscript, and to $\mathrm{J}$. O'Connell for her administrative and secretarial assistance.

This work was supported by the United Nations Developmental Program/World Bank/World Health Organization Special Programme for Research and Training in Tropical Diseases, the Veterans Administration, and National Institutes of Health grant AI-26505, and grant CNPq, SINEP (Brazil).

\section{References}

1. Weiler, I. J., E. Weiler, R. Springer, and F. Cosenza. 1977. Idiotype suppression by maternal influence. Eur. J. Immunol. 7:591597.

2. Wikler, M., C. Demeur, G. Dewasme, and J. J. Urbain. 1988. Immunoregulatory role of maternal idiotypes. J. Exp. Med. 152:1024-1033.

3. Bona, C. A. 1987. Regulatory idiotopes. In Modern Concepts in Immunology. Vol. 2. John Wiley \& Sons, NY. 279 pp.

4. Rubinstein, L. J., M. Yeh, and C. A. Bona. 1982. Idiotype-antiidiotype network. II. Activation of silent clones by treatment at birth with idiotypes is associated with the expansion of idiotype-specific helper T cells. J. Exp. Med. 153:506-521.

5. Kohler, H., J. Urbain, and P.-A. Cazenave. 1984. Idiotypy in Biology and Medicine. Academic Press, Orlando, FL. 445 pp.

6. Stein, K. E., and T. Soderstrom. 1984. Neonatal administration of idiotype or antidiotype primes for protection against Escherichia coli $\mathrm{K} 13$ infection in mice. J. Exp. Med. 160:1001-1011.

7. Kearney, J. F., and M. Vakil. 1986. Idiotype-directed interactions during ontogeny play a major role in the establishment of the adult B cell repertoire. Immunol. Rev. 94:39-50.

8. Lees, R. E. M., and P. Jordan. 1968. Transplacental transfer of antibodies to Schistosoma mansoni and their persistence in infants. Trans. R. Soc. Trop. Med. Hyg. 62:630-631.

9. Hillyer, G. V., R. Menendez-Corrada, R. Lluberes, and F. Hernandez-Morales. 1975. Evidence of transplacental passage of specific antibody in schistosomiasis mansoni in man. Am. J. Trop. Med. Hyg. 19:289-291.

10. Miles, M. A., V. Macedo, C. Castro, and C. C. Draper. 1975. Trypanosoma cruzi: Prenatal transfer of maternal antibody in man. Trans. R. Soc. Trop. Med. Hyg. 69:286. 
11. Sher, A., and D. G. Colley. 1989. Immunoparasitology: Chapter 35. In Fundamental Immunology. 2nd ed. W. E. Paul, editor. Raven Press, New York. In press.

12. Gelfand, M. 1967. A Clinical Study of Intestinal Bilharziasis (Schistosoma mansoni) in Africa. Edward Arnold (Publishers), Ltd., London. $230 \mathrm{pp}$.

13. Nash, T. E., A. W. Cheever, E. A. Ottesen, and J. A. Cook. 1982. Schistosome infections in humans: perspectives and recent findings. Ann. Intern. Med. 97:740-754.

14. von Lichtenberg, F. 1987. Consequences of infections with schistosomes. In The Biology of Schistosomes, From Genes to Latrines. D. Rollinson and A. J. G. Simpson, editors. Academic Press, London. 185-232.

15. Lima, M. S., G. Gazzinelli, E. Nascimento, J. Cavalho Parra, M. S. Montesano, and D. G. Colley. 1986. Immune responses during human Schistosomiasis mansoni. Evidence for antiidiotypic T lymphocyte responsiveness. J. Clin. Invest. 78:983-988.

16. Parra, J. C., M. S. Lima, G. Gazzinelli, and D. G. Colley. 1988. Immune responses during human schistosomiasis mansoni. XV. Anti-idiotypic $T$ cells can recognize and respond to anti-SEA idiotypes directly. J. Immunol. 140:2401-2405.

17. Gazzinelli, R. T., M. J. F. Morato, R. M. B. Nunes, J. R. Cançado, Z. Brener, and G. Gazzinelli. 1988. Idiotype stimulation of T lymphocytes from Trypanosoma cruzi-infected patients. J. Immunol. 14:3167-3172.

18. Gazzinelli, R. T., J. F. C. Parra, R. Correa-Oliveira, J. R. Cançado, R. S. Rocha, G. Gazzinelli, and D. G. Colley. 1988. Idiotypic/anti-idiotypic interactions in schistosomiasis and Chagas' disease. Am. J. Trop. Med. Hyg. 39:288-294.

19. Montesano, M. A., M. S. Lima, R. Correa-Oliveira, G. Gazzinelli, and D. G. Colley. 1989. Immune responses during human schistosomiasis mansoni. XVI. Idiotypic differences in antibody preparations from patients with different clinical forms of infection. J. Immunol. 142:2501-2506.

20. Colley, D. G., J. C. Parra, M. A. Montesano, M. Lima, E. Nascimento, B. L. Doughty, A. Goes, and G. Gazzinelli. 1987. Immunoregulation in human schistosomiasis by idiotypic interactions and lymphokine-mediated mechanisms. Mem. Inst. Oswaldo Cruz Rio J. 82(Suppl. IV):105-109.

21. Colley, D. G., A. M. Goes, B. L. Doughty, R. Correa-Oliveira, J. Parra, K. M. Lairmore, M. A. Montesano, R. S. Rocha, and G. Gazzinelli. 1988. Anti-idiotypic $T$ cells and factors in murine and human schistosomiasis. In Cellular Basis of Immune Modulation. J. G. Kap- lan, D. R. Green and R. C. Bleackley, editors. Alan R. Liss, Inc., NY. 367-378.

22. Boros, D. L., and K. S. Warren. 1970. Delayed hypersensitivity-type granuloma formation and dermal reaction induced and elicited by a soluble factor isolated from Schistosoma mansoni eggs. $J$. Exp. Med. 132:488-507.

23. Carter, C. E., and D. G. Colley. 1978. An electrophoretic analysis of Schistosoma mansoni soluble egg antigenic preparation. J. Parasitol. 64:385-390.

24. Morato, M. J., Z. Brener, J. R. Cançado, R. M. Nunes, E. Chiari, and G. Gazzinelli. 1986. Cellular immune responses of chagasic patients to antigens derived from different Trypanosoma cruzi strains and clones. Am. J. Trop. Med. Hyg. 35:505-511.

25. Nash, T. E., and A. M. Deelder. 1985. Comparison of four schistosome excretory-secretory antigens: Phenol sulfuric test active peak, cathodic circulating antigen, gut-associated proteoglycan, and circulating anodic antigen. Am. J. Trop. Med. Hyg. 34:236-241.

26. Santoro, F., Y. Carlier, R. Borojevic, D. Bout, P. Tachon, and A. Capron. 1977. Parasite " $M$ " antigen in milk from mothers infected with Schistosoma mansoni (preliminary report). Ann. Trop. Med. Parasitol. 71:121-122.

27. Camus, D., Y. Carlier, J. C. Bina, R. Borojevic, A. Prata, and A. Capron. 1976. Sensitization to Schistosoma mansoni antigen in uninfected children born to infected mothers. J. Inf. Dis. 134:405-408.

28. Tachon, P., and R. Borojevic. 1978. Mother-child relation in human schistosomiasis mansoni: skin test and cord blood reactivity to schistosomal antigens. Trans. R. Soc. Trop. Med. Hyg. 72:605-609.

29. Weil, G. J., R. Hussain, V. Kumaraswami, S. P. Tripathy, K. S. Phillips, and E. A. Ottesen. 1983. Prenatal allergic sensitization to helminth antigens in offspring of parasitic-infected mothers. J. Clin. Invest. 71:1124-1129.

30. Lewert, R. M., and S. Mandlowitz. 1969. Schistosomiasis: prenatal induction of tolerance to antigens. Nature (Lond.). 224:10291030.

31. Hang, L. M., D. L. Boros, and K. S. Warren. 1974. Induction of immunological hyporesponsiveness to granulomatous hypersensitivity in Schistosoma mansoni infection. J. Infect. Dis. 130:515-522.

32. Harte, P. G., and J. H. L. Playfair. 1983. Failure of malaria vaccination in mice born to immune mothers. II. Induction of specific suppressor cells by maternal IgG. Clin. Exp. Immunol. 51:157-164.

33. Gaulton, G. N., A. H. Sharpe, D. W. Chang, B. N. Fields, and M. I. Greene. 1986. Syngeneic monoclonal internal image anti-idiotopes as prophylactic vaccines. J. Immunol. 137:2930-2936. 